\section{Radiographic Findings and Clinical Correlates in Pediatric Periorbital Infections}

Keywords: Sinusitis; Subperiosteal abscess; Lund-McKay; Endosc opic sinus surgery; Periorbital c ellulitis

\begin{abstract}
Objective: To review radiographic studies of pediatric patients pre senting with periorbital infections and to evaluate sinonasal a natomical factors and clinical course related to this disease process.

Methods: Retrospective study review of computed tomography (CT) scans in 100 patients less than 18 years old, admitted to a tertiary children's hospital with the diagnosis of an orbital infection. CTscans were reviewed for anatomic variants and Lund-Mackay scores were calculated. An independent chart reviews of the treatment course and need for surgical intervention was performed.

Results: Of 100 patients, $67 \%$ were male, 60\% had left-sided infections, and $30 \%$ of patients were treated with surgical drainage. Ad enoid hypertrophy (61\%), inferior turbinate hypertrophy $(80 \%)$ and septal deviation (47\%) were common, but did not show statistic al correlation with the need for surgical intervention. Dehisc ence of the lamina papyracea was identified in $21 \%$ of patients treated without surgery and in $76 \%$ of those requiring surgery ( $P$ 0.0048). The average overall Lund-Mackay score was 11.8 and did not correlate with the need fo surgic al intervention.

Conclusions: To our knowledge, this is the first study to evaluate the incidence of sinonasal a na tomic abnomalities in children presenting with periorbital infections. This study also demonstrated that lamina papyracea dehiscence is a common finding and is associated with higher rates of surgical intervention. Such findings may have an important role in the diagnosis, surveillance and management of sinus disease in the pediatric population.
\end{abstract}

\section{Introduction}

Periorbital cellulitis (POC) presents with sudden onset of fever, erythema and swelling around the eye, decreased eye mobility, and possible vision loss. POC is a serious condition with multiple potential complications including orbital abscess formation and intracranial sequelae if untreated. As classified by Chandler, orbital complications can range from inflammatory edema to subperiosteal abscess, orbital abscess, cavernous sinus thrombosis, and other intracranial complications [1].

Most commonly, periorbital cellulitis in the pediatric population is associated with sinusitis [2]. The vast majority of patients can be successfully treated with antibiotics and other supportive measures; however, a subset of patients will require operative drainage. One of the more common and concerning complications of pediatric sinusitis is the development of an orbital subperiosteal abscess (SPA). The incidence of SPA as a complication of sinusitis is reported as approximately 9\% [3]. Potential anatomical pathways for spread of infection into the orbit include: 1) the anastamoses between the valveless venous network that drains the orbit, skin of the periorbital tissues, and the maxillary and ethmoid sinuses, 2) the thin, possibly

\author{
Jonathan M. Grischkan ${ }^{1,2}$, Charles A. Elmaraghy ${ }^{1,2}$, \\ Matthew R. Garrett ${ }^{1,2}$, Boris Karanfilov ${ }^{1,3}$ and \\ Kris R. Jatana ${ }^{1,2 *}$ \\ ${ }^{1}$ Department of Otolaryngology-Head and Neck Surgery, Wexner \\ Medical Center at Ohio State University, Columbus, Ohio, USA \\ ${ }^{2}$ Department of Pediatric Otolaryngology, Nationwide Children's \\ Hospital, Columbus, Ohio, USA \\ ${ }^{3}$ Ohio Sinus Institute, Columbus, Ohio, USA \\ *Address for Correspondence \\ Kris R. Jatana, M.D, Nationwide Children's Hospital, Otolaryngology- \\ Head and Neck Surgery, 700 Children's Dr, Columbus, $\mathrm{OH}$ \\ 43205,USA, Tel: (614) 722-6600; Fax: (614) 722-6609; \\ E-mail: Kris.Jatana@nationwidechildrens.org \\ Submission: 08 January 2015 \\ Accepted: 20 January 2015 \\ Published: 24 January 2015
}

dehiscent, lamina papyracea, 3) foramina of the ethmoidal arteries and 4) the orbital septum within the upper and lower eyelids, which is an extension of the periosteum [4].

The pediatric sinonasal anatomy has not been well investigated with respect to peri-orbital complications of sinusitis. In this study, we seek to describe the sinonasal anatomic characteristics and LundMackay (LM) scoring associated with periorbital infections and their relationship to the ultimate need for surgical intervention.

\section{Methods}

Approval was obtained from the Nationwide Children's Hospital Institutional Review Board for a retrospective study of medical records including radiographic studies. A consecutive review of patients admitted with a diagnosis of periorbital infection from 20032008 was performed. Inclusion criteria including the following:

1. Less than 18 years of age,

2. A CT study (orbits or paranasal sinuses) was obtained and available for review

\section{Inpatient admission for periorbital infection}

Within the review of consecutive patients, 20 patients were excluded from the study due to incomplete data, orbital trauma or foreign body, temporal space infections incorrectly coded as periorbital infections, and known orbital malignancy.

A total of 100 patient charts with complete computed tomography scans of the orbits or paranasal sinuses were available for review. The computed tomography scans were simultaneously examined by a fellowship-trained pediatric otolaryngologist and a fellowshiptrained rhinologist. The raters were blinded to all clinical details except the side of POC. Images were reviewed in at least axial and coronal planes, and saggital planes if available, and bone and soft tissue windows were analyzed individually. Individual data collected included age, gender, presence of adenoid hypertrophy, presence of middle turbinate abnormality such as pneumatization, lateralization or paradoxical position, and the presence of Haller cells. Inferior 
Citation: Grischkan JM, Elmaraghy CA, Garrett MR, Karanfilov B, Jatana KR. Radiographic Findings and Clinical Correlates in Pediatric Periorbital Infections. Inter J Otorhinolaryngology. 2015;2(1): 5.

turbinate hypertrophy was also marked as present or absent, and lamina papyracea dehiscence was noted to absent or present.

All scans were scored according to the standard Lund-Mackay system. Each paranasal sinus was graded on standard scales of 0,1 or 2 for absence of opacification, partial, or complete opacification respectively. Total individual scores may range from 0-24. If any of the paranasal sinuses such as the frontal or sphenoid were deemed to be undeveloped, a score of 0 was assigned for that sinus.

In addition, the clinical course of each subject was separately and independently reviewed. Data points were collected with regards to the need for surgical intervention, findings at the time of surgery, and microbiological results from intra-operative collections. In addition, subjects were analyzed with respect to the use of antibiotics preadmission.

Data were tabulated from individual scoring sheets and statistical analysis performed. All data points were independently described and correlated to the need for surgical intervention. Chi-square test or Fisher's Exact test were used appropriately to test distribution of categorical variables between surgical and nonsurgical patients. T-test was used to compare total LM score between surgical and nonsurgical patients. All tests were conducted using SAS 9.2.

\section{Results}

Mean age of the patients included in the study was approx 6.8 years (81.5 months). Patients treated surgically were on average slightly older with an average age of 92 months versus 76 months in the non-surgery group ( $\mathrm{p} 0.226$ ). There were a total of 67 males and 33 females. POC was slightly more frequent on the left (63\%), but was not statistically significant. Out of 100 patients, 30 underwent surgery as part of their management. Age, side, and gender were not associated with the ultimate need for surgical intervention (Table 1).

Radiographic anatomic findings for all patients included a high percent of adenoid hypertrophy (61\%), inferior turbinate hypertrophy (80\%) and septal deviation (47\%) in the both the surgical and non-surgical group. Adenoid hypertrophy and inferior turbinate hypertrophy were graded as present or absent (Figure 1). The nasal septum was graded as mid-line or deviated. Deviation was noted as ipsilateral or contralateral with respect to the side of infection. Thirty two patients (32\%) had septal deviation ipsilateral to the side of their infection, and 15 (15\%) had septal deviation contralateral to the side of infection. Adenoid hypertrophy, inferior turbinate hypertrophy, and nasal septal deviation did not correlate with surgical intervention.

Haller cells, middle turbinate abnormalities, and uncinate abnormalities were uncommon. Haller cells were present in $9 \%$ of all patients (Figure 2). Pneumatization of the middle turbinate was present in $13 \%$ of all patients. Lateralization of the middle turbinate was not seen in any of the patients. Paradoxical middle turbinates were noted in $5 \%$ of the patients. The presence of Haller cells and middle turbinate abnormalities was not associated with surgical intervention.

Lamina papyracea dehiscences were graded as absent or present (Figure 3). Dehiscence demonstrated statistical significance between surgical and non-surgical groups. Dehiscence was detected in 57\%
Table 1: Clinical and anatomic findings in non-surgical and surgical patients.

\begin{tabular}{|c|c|c|c|}
\hline & $\begin{array}{l}\text { Non-Surgical } \\
(n=70)\end{array}$ & Surgical $(n=30)$ & $P$ value \\
\hline $\begin{array}{l}\text { Age (mean) } \\
\text { Gender }\end{array}$ & $\begin{array}{c}76 \text { months } \\
45 / 70 \text { male }(64 \%)\end{array}$ & $\begin{array}{c}92 \text { months } \\
22 / 30 \text { male }(73 \%)\end{array}$ & $\begin{array}{l}0.23 \\
0.43\end{array}$ \\
\hline Left side infection & $63 \%$ & $53 \%$ & 0.37 \\
\hline Adenoid hypertrophy & $63 \%$ & $57 \%$ & 0.56 \\
\hline Septal deviation & $47 \%$ & $53 \%$ & 0.53 \\
\hline Inferior turbinate hypertrophy & $76 \%$ & $90 \%$ & 0.10 \\
\hline Haller cell & $10 \%$ & $7 \%$ & 0.27 \\
\hline Middle turbinate abnormality & $16 \%$ & $23 \%$ & 0.60 \\
\hline Uncinate abnormality & $0.1 \%$ & $3 \%$ & 0.51 \\
\hline $\begin{array}{l}\text { Lamina papyracea } \\
\text { dehiscence }\end{array}$ & $27 \%$ & $57 \%$ & 0.0048 \\
\hline Pre-admission antibiotics & $44 \%$ & $60 \%$ & 0.15 \\
\hline Lund-Mackay scores & $12.4(\mathrm{SD} 5.7)$ & $11.7(\mathrm{SD} 5.1)$ & 0.56 \\
\hline
\end{tabular}

$\mathrm{SD}=$ standard deviation

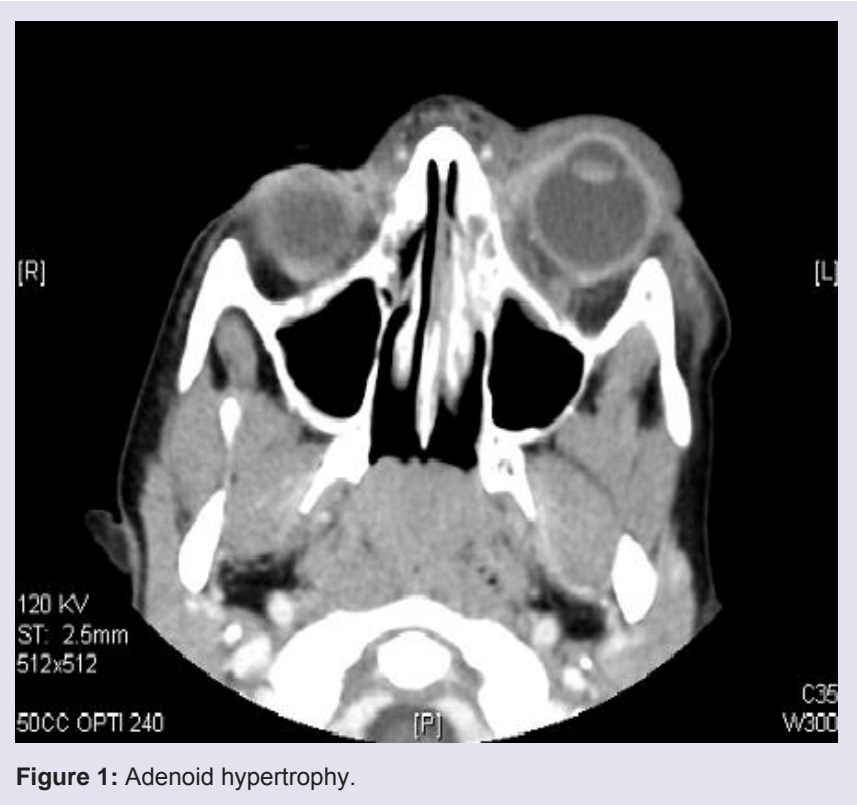

of patients who ultimately were treated with surgery, and in $27 \%$ of those treated without surgery ( $p .0048)$.

LM scores were also calculated for each patient. Scores were not different between surgical and non-surgical patients. Both groups demonstrated a high degree of sinus opacification. The mean scores were 11.7 and 12.4 with a standard deviation of 5 and 5.7 for the surgical and non-surgical groups respectively (P 0.56), and in the non-surgical group, scores ranged from $0-23$ and in the surgical group from 2-21. Both groups showed a wide range of sinus opacification at the time of initial imaging.

Approximately half of all patients (49\%) were initially treated with antibiotics in an ambulatory setting prior to their admission. The use of antibiotics pre-admission did not correlate with the ultimate need for surgical treatment. (P 0.15) 
Citation: Grischkan JM, Elmaraghy CA, Garrett MR, Karanfilov B, Jatana KR. Radiographic Findings and Clinical Correlates in Pediatric Periorbital Infections. Inter J Otorhinolaryngology. 2015;2(1): 5.

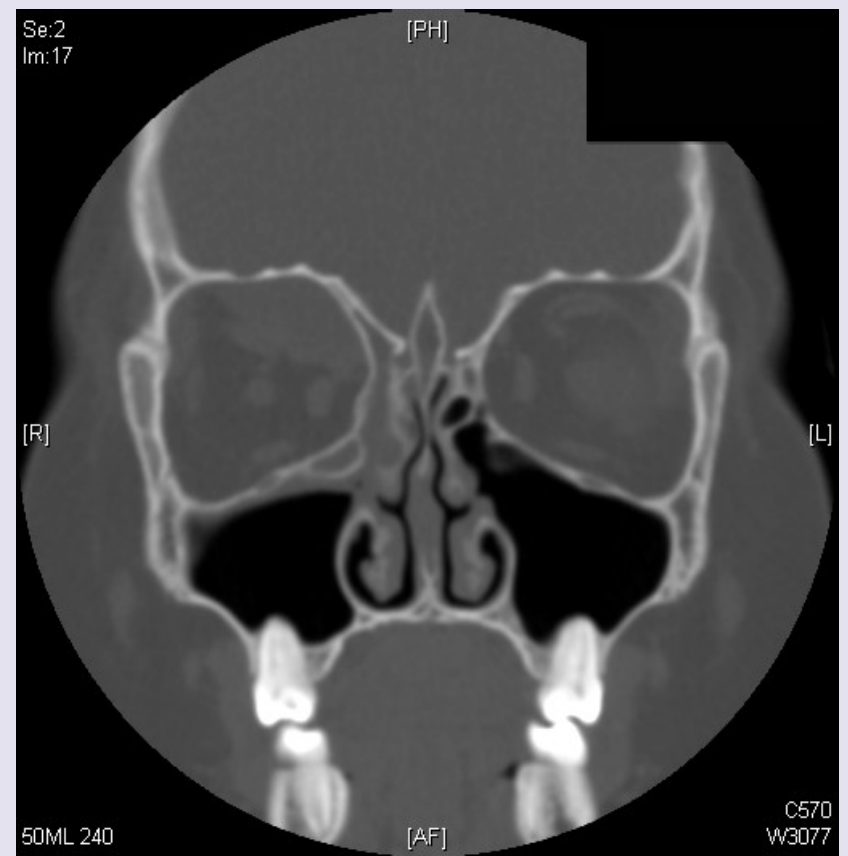

Figure 2: Haller cell present on side of infection.

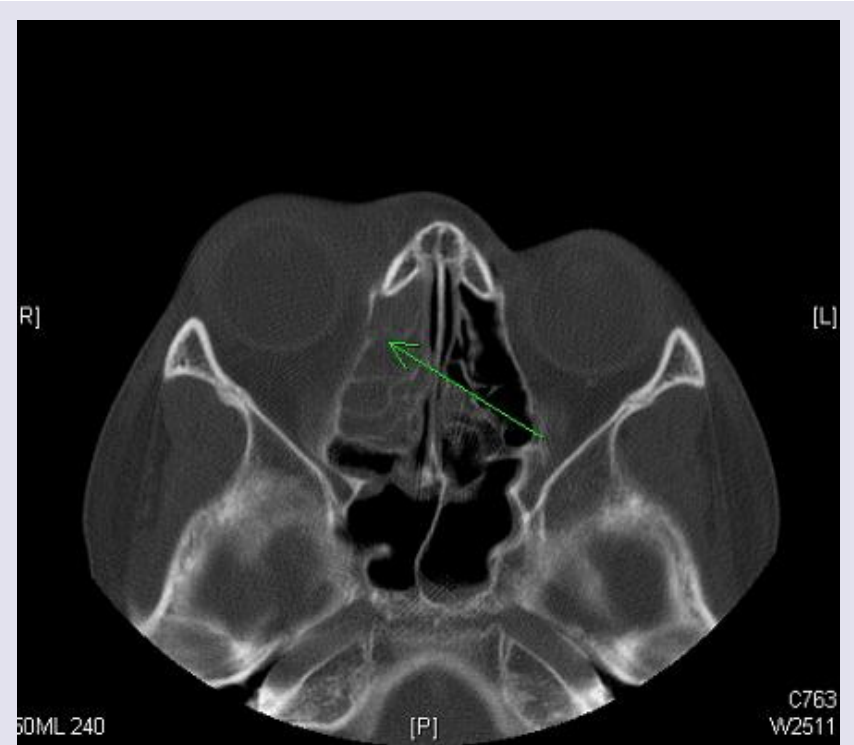

Figure 3: Demonstration of lamina papyracea dehiscence.

Microbiological samples were taken at the time of surgery. Out of 30 patients, 10 had negative cultures, 4 patients grew Staphylococcus aureus, 3 grew Hemophilus influenzae, 3 grew Group A strep, and the remainder were reported as "normal respiratory flora" without a dominate organism.

\section{Discussion}

To our knowledge, this is the first study to review the radiographic sinonasal anatomy of pediatric patients presenting with periorbital infections. We noted a high degree of sinus mucosal disease, shown by the large proportion of patients with LM scores greater than 10 .
The anatomic abnormalities noted on the scans, including adenoid hypertrophy, inferior turbinate hypertrophy and septal deviation were common. We also demonstrate that severity of sinusitis based on LM scoring does not correlate with the ultimate need for surgical intervention.

Interestingly, the raters were able to identify a dehiscence in the lamina papyracea on the side of disease in approximately $1 / 3$ of the radiographs examined, suggesting that the presence of this anatomic abnormality may play a significant role in the development of periorbital infection. Although the association between the dehiscent lamina and the overall LM score did not reach statistical significance, there was statistically significant association between lamina dehiscence and the ultimate need for surgery. Approximately one third of all patients reviewed in this study demonstrated evidence of lamina papyracea dehiscence (36\%). Those who ultimately required surgery demonstrated a dehiscence rate of $76 \%$.

We feel that this may be an important contributing factor in the orbital complications seen with pediatric sinusitis and may ultimately be a harbinger of the need for surgical intervention. Given the descriptive correlation provided by the radiographic and clinical review and the fact that only patients acutely presenting with POC were reviewed, it is uncertain what the rate of dehiscence would be in the non-acute setting or in patients with sinusitis but without periorbital complications. It may be that acute sinusitis enlarges, creates, or "reveals" a subclinical dehiscence. It is also possible that patients with a pre-existing radiographically detectable dehiscence are more likely to develop periorbital complications in the setting of acute sinusitis. As previously noted, paranasal sinus infections can involve the orbit via several routes. The majority of patients with orbital involvement did not have radiographically detectable evidence of lamina dehiscence, suggesting that other routes of spread such as local thrombophlebitis or infected thromboemboli also play an important role [4-6].

A recent report by Ryan described an incidence of SPA of $28 \%$ in a series of 240 CT scans obtained for POC [7]. Out of 68 patients who were found to have SPA, 21 ultimately required surgery. In their study, surgical intervention was statistically more likely in patients with larger abscesses, higher temperatures, and older age. In the present study, we report a higher rate of surgical intervention (30\%). Our study did show a slight trend towards surgery in the older patient (6.3 vs 7.7 years), but was not statistically significant. Multiple other studies have also demonstrated that younger patients are more likely to have less complicated infections and more likely to respond to medical therapies [8-10].

The ultimate need for surgical intervention in our series was based upon the clinical presentation and the clinical course of each patient. All patients underwent ophthalmological evaluation and were treated with intravenous antibiotics beginning at the time of admission. Multiple factors determined the need for surgery including the severity of the initial presentation, radiographic findings, ophthalmologic evaluation, and the response to aggressive medical management including intravenous antibiotics, topical and systemic nasal decongestants. Surgery for patients in this study typically entailed an endoscopic approach with a complete uncinectomy, maxillary antrostomy, anterior ethmoidectomy and lamina paparecia 
Citation: Grischkan JM, Elmaraghy CA, Garrett MR, Karanfilov B, Jatana KR. Radiographic Findings and Clinical Correlates in Pediatric Periorbital Infections. Inter J Otorhinolaryngology. 2015;2(1): 5.

decompression. Intraoperative stereotactic CT guidance was used for all cases.

Acute sinusitis is well recognized as the etiology of many cases of periorbital infections, but no previous study has analyzed the paranasal sinus anatomy with respect to periorbital infections. Specifically, the use of LM scoring has not been previously studied. A previous radiographic study by Moubayed et al. looked at 117 patients with a diagnosis of POC [11]. In their review, $24.8 \%$ of periorbital infections were attributed to a sinusitis etiology. Other common etiologies included trauma, skin infections, and ocular infections. In their series, maxillary and ethmoid disease was equally common which reflects the distribution of sinus involvement in our study, but we report a much higher degree of sinusitis. Work by Bhattacharyya et al. demonstrated that LM scoring has high sensitivity and specificity for true sinus disease in the pediatric population [12]. In their study, pediatric patients with known chronic rhinosinusitis at an average age of 8 years demonstrated LM scores of 10.4 versus nondiseased age-match controls which demonstrated average scores of 2.8. Additional work has confirmed that the incidental LM score in non-disased pediatric patients in this age range is 3 [13]. In our study, a high degree of sinus disease was present. The average LM score was 12.3 (SD 5.5) with similar numbers in the surgical and non-surgical group.

Anatomic variants are also thought to play a role in development of sinusitis. Uncinate abnormalities have been reported at the time of endoscopic drainage of SPAs [14,15]. The role of adenoid hypertrophy in pediatric sinusitis has been extensively discussed in previous publications and is beyond the scope of this discussion $[16,17]$. Our present study demonstrates a high rate of adenoid hypertrophy (61\%). This likely reflects the age demographic and the acute illness of the patients included in this study. Previous authors have reached differing conclusions regarding the role of other bony sinus abnormalities such as concha bullosa, Haller cells, and nasal septal deviation to the development of mucosal sinus disease [1821]. These variants potentially lead to obstruction of the osteomeatal complex and downstream effects on anterior ethmoid drainage. In the present study, the osteomeatal complex was graded during LM scoring. Both surgical and non-surgical groups had a high degree of obstruction with 129 of 200 OMCs reviewed demonstrating evidence of obstruction.

Previous work has also demonstrated that patients who are not treated with antibiotics prior to admission are more likely to require surgery [22]. These authors hypothesized that those patients who did not receive antibiotic therapy prior to admission represented a subset where treatments were commenced too late in the course of illness to prevent SPA. They suggest that SPA may be mitigated by early antibiotic therapy. However, they also point out, that the majority of patients present with less than 4 days of total symptoms, and that the American Academy of Pediatrics recommends 3-4 days of high fever and purulent nasal discharge to define the presentation of severe acute bacterial sinusitis for which antibiotics are indicated [23]. This in turn may mean that many patients present with orbital complications before antibiotics have been given for preceding sinusitis symptoms. In our present study, roughly half of all patients were treated with antibiotics prior to presentation and admission. This did not appear to be related to the ultimate need for surgical intervention.

This study is limited by its retrospective nature. Patients were sampled at one point in time during an acute infectious process. There is no true negative control. The anatomic variations in a cohort of pediatric patients without a history of sinusitis or peri-orbital infections were not analyzed. This study did not correlate the size of abscess or the duration of hospitalization, which are important factors in management strategies. There is also a bias toward increased severity of disease given that only those patients with severe enough symptoms to warrant a CT and admission to the hospital were reviewed.

\section{Conclusion}

Periorbital complications of sinusitis represent a serious clinical scenario and may lead to significant morbidity if not promptly treated. Significant mucosal sinus disease did not correlate with increased need for surgical drainage. This study demonstrates that lamina papyracea dehiscence is a common finding and is associated with higher rates of surgical intervention. Such findings may have an important role in the diagnosis, surveillance and management of sinus disease in the pediatric population.

\section{References}

1. Chandler JR, Langenbrunner DJ, Stevens ER (1970) The pathogenesis of orbital complications in acute sinusitis. Laryngoscope 80: 1414-1428.

2. Israele V, Nelson JD (1987) Periorbital and orbital cellulitis. Pediatr Infect Dis J 6: 404-410.

3. Sobol SE, Marchand J, Tewfik TL, Manoukian JJ, Schloss MD (2002) Orbita complications of sinusitis in children. J Otolaryngol 31: 131-136.

4. Jackson K, Baker SR (1986) Clinical implications of orbital cellulitis Laryngoscope 96 : 568-574.

5. Wiatrak BJ (1999) Orbital subperiosteal abscess. In: Cotton R, Myer C, editors. Practical Pediatric Otolaryngology. Philadelphia (PA): LippincottRaven p. 978-982.

6. Wald ER (2007) Periorbital and orbital infections. Infect Dis Clin North Am 21: 393- 408.

7. Ryan JT, Preciado DA, Bauman N, Pena M, Bose S, et al. (2009) Management of pediatric orbital cellulitis in patients with radiographic findings of subperiosteal abscess. Otolaryngol Head Neck Surg 140: 907-911.

8. Oxford LE, McClay J (2006) Medical and surgical management of subperiosteal orbital abscess secondary to acute sinusitis in children. Int $J$ Pediatr Otorhinolaryngol 70: 1853-1861.

9. Garcia GH, Harris GJ (2000) Criteria for nonsurgical management of subperiosteal abscess of the orbit: analysis of outcomes 1988-1998. Ophthalmology 107:1454-1456

10. Starkey CR, Steele RW (2001) Medical management of orbital cellulitis. Pediatr Infect Dis J 20: 1002-1005

11. Moubayed SP, Vu TT, Quach C, Daniel SJ (2011) Periorbital cellulitis in the pediatric population: clinical features and management of 117 cases. J Otolaryngol Head Neck Surg 40: 266-270.

12. Bhattacharyya N, Jones DT, Hill M, Shapiro NL (2004) The diagnostic accuracy of computed tomography in pediatric chronic rhinosinusitis. Arch Otolaryngol Head Neck Surg 130: 1029-1032.

13. Hill M, Bhattacharyya N, Hall TR, Lufkin R, Shapiro NL (2004) Incidenta paranasal sinus imaging abnormalities and the normal Lund score in children. Otolaryngol Head Neck Surg 130: 171-175. 
Citation: Grischkan JM, Elmaraghy CA, Garrett MR, Karanfilov B, Jatana KR. Radiographic Findings and Clinical Correlates in Pediatric Periorbital Infections. Inter J Otorhinolaryngology. 2015;2(1): 5.

14. Karkos PD, Karagama Y, Karkanevatos A, Srinivasan V (2004) Recurrent periorbital cellulitis in a child: a random event or an underlying anatomica abnormality? Int J Pediatr Otorhinolaryngol 68: 1529-1532.

15. Jatana KR, Grischkan JM, Skomorowski MJ, Elmaraghy CA (2008) Recurrent unilateral periorbital cellulitis in a pediatric patient--an anatomic abnormality. Int J Pediatr Otorhinolaryngol 72: 1577-1580.

16. Ramadan HH, Cost JL (2008) Outcome of adenoidectomy versus adenoidectomy with maxillary sinus wash for chronic rhinosinusitis in children. Laryngoscope 118: 871-873.

17. Ramadan $\mathrm{HH}$, Tiu J (2007) Failures of adenoidectomy for chronic rhinosinusitis in children: for whom and when do they fail? Laryngoscope 117 1080-1083.

18. Nouraei SA, Elisay AR, Dimarco A, Abdi R, Majidi H, et al. (2009) Variations in paranasal sinus anatomy: implications for the pathophysiology of chronic rhinosinusitis and safety of endoscopic sinus surgery. J Otolaryngol Head Neck Surg 38: 32-37.
19. Caughey RJ, Jameson MJ, Gross CW, Han JK (2005) Anatomic risk factors for sinus disease: fact or fiction? Am J Rhinol 19: 334-339.

20. Aktas D, Kalcioglu MT, Kutlu R, Ozturan O, Oncel S (2003) The relationship between the concha bullosa, nasal septal deviation and sinusitis. Rhinology 41: 103-106

21. Bolger WE, Butzin CA, Parsons DS (1991) Paranasal sinus bony anatomic variations and mucosal abnormalities: CT analysis for endoscopic sinus surgery. Laryngoscope 101: 56-64.

22. Sinclair CF, Berkowitz RG (2007) Prior antibiotic therapy for acute sinusitis in children and the development of subperiosteal orbital abscess. Int J Pediatr Otorhinolaryngol 71: 1003-1006.

23. American Academy of Pediatrics. Subcommittee on Management of Sinusitis and Committee on Quality Improvement (2001) Clinical practice guideline: management of sinusitis. Pediatrics 108: 798-808.
Wei Wang: Statistical review of data. The project described was supported by Award Number Grant UL1TR001070 from the National Center For Advancing Translational Sciences. The content is solely the responsibility of the authors and does not necessarily represent the official views of the National Center For Advancing Translational Sciences or the National Institutes of Health. 